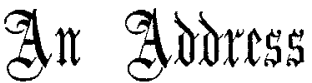

on

\section{THE TREATMENT OF PHTHISIS.}

Being some Remarks, introductory to a Discussion, delivered at the Harveian Society on Dec.8th, 1904,

By HECTOR W. G. MACKENZIE, M.D. Cantab., F.R.C.P. Lond.,

PHYSICIAN TO ST. THOMAS'S HOSPITAL AND TO THE BROMPTON HOSPITAL FOR CONSUMPTION.

Mr. Chairman and Gentlemen,-The subject of the discussion to-night-the treatment of phthisis-is one which every physician and every general practitioner has again and again to consider and to advise upon. It may be the case that we in this society are all at one as to the methods to be employed and the results to be obtained and that after the many discussions which have been held there is nothing more to be added to our knowledge of the subject. We cannot, however, shut our eyes to the fact that, although there may be perfect unanimity here in our midst, there are many members of the profession who do not at heart believe in what is called open-air treatment and look upon it as something of the nature of a passing craze. Indeed, I am told that at the present time as regards sanatorium treatment a kind of reaction has set in which is very likely due to the fact that some enthusiastic propagandists put forward exaggerated and extravagant claims as to what these institutions could do. The ever-credulous public were led to believe that by means of sanatorium treatment consumption could be cured irrespective of the stage of the disease or the state of the patient. When they find that the patient is given air and is not soon well then they disparage the mode of treatment and pass an unjustly unfavourable judgment upon it. In this age of claptrap and catchpenny with its sensational journalism of seeming cheapness we hear more and more of new cures for consumption of which several are prematurely announced in the course of the year. The cure of consumption will always attract quacks for several reasons. Patients who have never had the disease will be cured and some undoubtedly affected by the disease will improve or actually get well while being treated by almost any remedy not absolutely lethal. In the case of patients who do not do well it is easy to allege that they have come too late and that a cure might have been effected had the remedy been tried at an earlier period in the disease. A harvest may be reaped before the real uselessness of a particular mode of treatment has been demonstrated. At a large consumption hospital such as Brompton any rational form of treatment can be thoroughly tested. When a cure is first exploited as a means of making money its genuineness is ipso facto very much to be doubted.

I propose, then, it is needless to say, only to consider such methods of treatment as have been thoroughly and efficiently tried. With present knowledge one must place in the foremost rank as remedies pure air and sunshine, good food, suitable raiment, and proper housing, with regulated rest and exercise under medical supervision. The most marked advance in the treatment of pulmonary phthisis and one which I believe will have far-reaching effect on the health of the human race is the recognition of the vital importance of fresh air to breathe. Fresh air essential for the maintenance of health is one of the most powerful factors in curing disease. We all know how much more attention was formerly paid to keeping up the temperature of the air indoors than to maintaining its purity. In their homes the consumptive invalids kept their windows religiously closed and shunned the open window as they would the plague. In hospitals elaborate systems of artificial ventilation have been employed with the object of supplying pure air at a comfortable temperature. These have all proved unsatisfactory hitherto.

The prevailing unwholesome dread and dislike of the open window is indirectly responsible for much of the existing pulmonary disease, so commonly attributed to our uncertain climate. Fear of fresh air has been the direst enemy of the consumptive invalid. The worst air for breathing is air which has been vitiated by the organic impurities given of in the course of respiration. The only way to obtain anything like an adequate degree of freshness in the air for an invalid indoors is to have the windows and preferably the door also widely open. Dust, smoke, and fog in the air are undesirable, and on account of them the air of towns is less wholesome than that of the country, but it is absurd and ludicrous to speak as some do of the air of London getting used up and becoming unfit to breathe towards the end of the London season. It is our object at the present day to make it possible for our consumptive patients constantly to obtain air to breathe as nearly as possible the same as the outside air. At the Brompton Hospital we now rely entirely on natural ventilation by means of open windows and open doors and the benefit to the patients is, I think, very apparent. At St. Thomas's Hospital we have for several years past used our balconies for open-air treatment, patients remaining there in their beds during the night as well as the day with excellent results. Where there is space available an outdoor shelter is a valuable auxiliary to treatment and a patient in a shelter or on a balcony properly protected from the elements will get a more abundant supply of fresh air than in even the airiest bedroom.

The advantages of the open-air treatment may be thus briefly summarised. The patient exposed continuously to fresh air gains in appetite, assimilates his food better, sleeps more soundly, and awakens more refreshed. Free exposure to air is the best antipyretic. Sweating at night, formerly so common a symptom, usually ceases. Colds are practically unknown among patients leading an open-air life. Secondary infection on account of the comparative freedom of the air from micro-organisms is much less likely to occur. Tolerance of outside air is very quickly established and no one who has tried the open-air life will willingly go back to the former conditions of stuffiness. I have never seen anyone made worse by exposure to fresh air. Even during a thick London fog patients get on better lying in bed on a balcony or in rooms with windows widely open and a good fire burning than when attempts are made to shut out the fog by keeping the windows shut.

On the beneficial influences of sunlight and sunshine on the tissues of the body it is unnecessary to enlarge. Among them are improvement in the quality and increase in the oxygenating power of the blood, stimulation of metabolism, and quickening of the activity of the nervous system. We should endeavour to secure for our patients the full advantages of all available sunlight and sunshine, especially during the winter months. Of paramount importance in treatment is the feeding of the patient. The building up of the tissues of the body and the repair of the waste are dependent on adequate nourishment. A falling.off of the appetite is an almost constant symptom of the disease and not the least benefit brought about by the open-air life is an increased desire for food. Much has been heard of the socalled overfeeding indulged in at some sanatoriums. Save in very exceptional cases anthing like stuffing is quite unnecessary. At first the patient must be encouraged to eat, not because he feels hungry or inclined for food, but for the reason that feeding is essential for overcoming the disease. He should be told to take plenty of time over his food, eating it slowly and thoroughly masticating it. As a rule, both an increase in the quantity and an improvement in the quality of the food is needed. The food should be wholesome, nutritious, and well-cooked, and served in an appetising way, dishes which should be hot being hot, not lukewarm. Three good meals a day should be given and nothing during the intervals between. 'There is often a distaste for fat which is a most important element of the food for the phthisical and the patient should be encouraged to take bacon, butter, cream, \&c., in fair quantity.

One of the most remarkable features of pulmonary phthisis is the capacity which the subjects of the disease have for putting on flesh. I have found it a much harder matter to fatten a thin, non-tuberculous subject than one who is tuberculous. Care must be taken, indeed, that the patient does not put on flesh too rapidly or to excess. In regulating the amount of food due regard must be paid to the state of nutrition. The thin, underweight patient requires a larger relative amount than the patient who is already up to his proper weight. Alcobol has been considered by some as one of our chief remedial agents and by others as not only unnecessary but harmful. The well-known case related long ago by Dr. Stokes of Dublin of the man who recovered from phthisis 
(every local and general symptom occurring in the highest degree) after consuming seldom less than seven tumblers of whiskey punch per diem has been often quoted. But the man while he was not drinking punch spent his time in the open air, fishing or duck shooting, and, as Dr. Walshe says, the case only serves to show that where the disposition to repair is strong even outrageous abuse of alcohol will not counteract or even injuriously modify that disposition. The frequent incidence of phthisis in cases of chronic alcoholism, of which there is no manner of doubt, is convincing proof that alcohol is at any rate powerless to prevent the inroads and progress of tuberculosis on the pulmonary system. If this be so, how can it reasonably be maintained that alcohol can have any curative value? But I look on alcohol as a luxury in cases where an adequate amount of nourishment is being taken. It is, however, a valuable medicine in acute cases and in the later stages of the disease.

The clothing should be sensible and properly adapted to the season of the year. At all times too much wrapping is as much to be avoided as too little and is, perhaps, the more common and the more serious error. As a rule, there is no difficulty in keeping patients warm while confined to bed however cold the weather and however free the exposure to the air. It is principally a matter of sufficiency of blankets, which should not, however, be too heavy. When necessary, warmth can be provided by hot-water bottles and when the thermometer is low the hands may be protected by woollen gloves, the head by a Balaclava helmet, and the feet by woollen socks. In the case of patients who are up overcoats, wraps, and rugs should be employed only when the patient is resting. The use of mufflers and chest protectors should not be encouraged. While in the matter of housing fresh air and sunshine are our first considerations, comfort should not be disregarded. The rooms occupied by the patient should be fairly large and, if possible, have a southerly exposure so as to secure the maximum of light and sunshine. Everything in the rooms should be kept fresh and clean, damp dusters being employed for the removal of dust. Unnecessary articles of furniture, thick curtains, and hangings should not be permitted to remain. In private houses open fires are necessary for comfort and in institutions where radiators are employed for heating it is a great adrantage to have open fires also.

No less important than air and food as remedial agents are rest and exercise, and the regulation of the form and proper proportion of these is one of the principal duties of the medical attendant. Our chief guide in the matter of rest is the temperature. When there is continued pyrexia absolute and complete rest is essential and this holds also where the evening temperature is $101^{\circ} \mathrm{F}$. or more, although the morning temperatures are not above normal. The morning temperature as a rule should determine whether the patient is to get up or not. When it is above normal the patient will remain in bed instead of getting up. The regulation of rest by the degree of temperature brings one to the debatable point of the relative value of axillary, oral, and rectal temperatures. There is no doubt that the rectal temperature is the most trustworthy index of the temperature of the body but except in special cases $I$ see no advantage to be gained by taking the temperature in the rectum. The condition of the pulse is another factor in deciding as to rest or exercise. Patients with a pulse-rate persistently over 100 should be kept at rest. On the whole, however, the rate of the pulse is much less important than the temperature.

The plan of making patients who are up all day rest in the recumbent position for half an hour before the two principal meals is one which has been found in practice to be very beneficial. Exercise must be proportioned to the strength and capabilities of the patient. The best form of exercise for most patients, because it can be thoroughly controlled by the physician, is walking. The length of the walk, its direction, and its rate will be adjusted to the individual case. The longer walk of the day should be taken shortly after breakfast. The length will vary from half a mile to five or six miles and the rate from one and a half to three miles an hour. If the country is billy it should be arranged that the patient goes uphill and returns downhill. All walks uphill should be taken slowly and deliberately, and the patient should always rest when he feels he is getting out of breath. In the afternoon a shorter walk may be taken. The effect of exercise on the temperature of the healthy body is temporarily to raise it one or two degrees. When the exercise is excessive the temperature will be found to have risen more than two degrees. The taking of the temperature after exercise is a valuable check on any excess. Over-exertion is very harmful and all forms of violent exercise must be emphatically vetoed. Cycling, skating, swimming, running, riding, fencing, dancing, tobogganing, cannot safely be permitted to anyone with signs of active disease. In cases where the disease is arrested such exercises as riding, golf, and cycling in moderation are beneficial.

Whether a patient should be treated at home or elsewhere depends on the nature of the case, on the home conditions, and on ways and means. The question of ways and means is often of much importance. It may sometimes be considerably less expensive for the patient to go to a sanatorium than to remain at home. It may be impossible at home to get proper attention. The home conditions may be altogether unsatisfactory. But where the home conditions are good where the patient can have a clean, light, airy room and good food and attendance, home treatment has considerable advantages in all cases where it is necessary to keep the patient continuously in bed. From my experience of cases treated on our balconies at St. Thomas's Hospital or in our airy wards at Brompton Hospital I should say emphatically that the results compare favourably with those of any country sanatorium in the kingdom. Therefore I am of opinion that if the home conditions are good it is often best to keep the patient at home during the continuance of pyrexia. Often, however, home conditions are entirely harmful and then no hesitation should be felt in recom. mending the removal of the patient to a suitable institution.

The advantages which sanatorium treatment presents are many. The patient is under constant medical supervision. $\mathrm{He}$ is removed from all disturbing influences; he is encouraged to take adequate amounts of food; he is made to take sufficient rest; he is prevented from over-exertion; he is induced to take the proper amount of exercise; he is taught how to live; he breathes pure air continuously throughout the day and night; he lives with one object, that of getting well; and he has a constant object lesson in watching the good effects of the treatment on others. No one who is well enough to be up and about can fail to derive the greatest benefit from residence at a sanatorium. He is able to take his walks among woodlands and country lanes and if he is a lover of nature he cannot but find much to interest him during his rambles. It must be clearly understood that satisfactory results are only obtained where plenty of time is given to the cure and that treatment must be carried out faithfully for at least six months and often for a much longer time in order to obtain satisfactory results. It must be confessed that the proportion of absolute cures is not very large, if by absolute cure is meant the disappearance of symptoms and of abnormal physical signs, together with absence of bacilli from the sputum. We must be content in most cases with a relative cure where symptoms are in abeyance and signs of activity of disease disappear.

I am strongly of opinion that for a large class of patients. sanatorium treatment affords the best chance of cure which we can offer at present. I must, however, protest against the lack of judgment sometimes shown in the selection of cases for this mode of treatment. It is no use sending advanced and actively progressing cases. Such cases are apt to bring discredit on the treatment and their presence in a sanatorium has a bad influence on the other patients. In deciding as to sanatorium treatment we should take into account the temperament and social position of the patient as well as the stage of the disease. To every patient whom one recommends to go to a sanatorium it may be said: "I cannot possibly promise that your treatment there will cure you or even benefit you but I can honestly tell you that in this way you will be giving yourself the best chance of battling with the disease successfully."

The effects of climate per $s e$ are difficult to distinguish from the effects of the conditions under which the patient has to live. At most health resorts, both at home and abroad, hotel life is utterly unsuitable for consumptive invalids. We all know the intolerably stuffy atmosphere of the public rooms and passages at some of the best hotels. Where a patient can afford to have his own suite of rooms and have his meals served in his own apartments it is a different matter. In most cases a sanatorium, or villa, or flat, or private apartments should, as a rule, be selected in preference to a hotel. 'The good results obtained by sanatoriums in many different parts of England go far to show that our home climate is not unfavourable to lung patients and that. 
even in winter residence in England is quite compatible with an open-air life. But who that can do so would not exchange our dull English winter and spring for the bright sunshine of the lofty Swiss valleys or the French and Italian Riviera? The pure, dry, calm air of Davos or St. Moritz, with many bright and sunny days throughout the winter, I consider to be of the highest value. St. Moritz has become of late much frequented by smart society people in search of pleasure. Its winter climatic advantages are, however, quite as great as those of Davos. For chronic quiescent cases the climate is excellent but there are so many temptations even at Davos to over-exertion and various imprudences that only those who can be trusted to take care of themselves should be allowed to go there except to a sanatorium. Sea voyages I find are still often recommended to consumptive patients by general practitioners as well as by physicians. I have seen some good results but I have seen some most disastrous ones. I cannot too strongly condemn the indiscriminate advice to patients to go for a sea voyage. The conditions on board ship are very far from those we desire for our patients. The deck is all right but the cabin is all wrong and in the event of the patient being taken ill on the way and confined to his berth the conditions are most unfavourable. No patient in whom there is any sign of active disease should be permitted to take a sea voyage for health. Even in quiescent cases much harm is done. I know of two quiescent cases where the passage through the Red Sea was attended by renewed activity and the patients were put ashore at Colombo and sent home to die.

Our mode of treatment often requires modification in individual cases. The condition of the digestive organs sometimes presents a formidable difficulty but it is remarkable how comparatively seldom one meets with any really serious dyspeptic trouble among phthisical patients. Indifference or actual repugnance to food is the most common trouble and, as a rule, the worse the nutrition and the greater the need of food the less inclination there is for it. This must be overcome by persuasion and suggestion, assisted by a bitter alkaline or acid tonic. In some cases coughing is induced by the presence of food in the stomach and the cough may continue until vomiting occurs and the contents of the stomach are expelled. In such cases the patient must be enjoined to keep perfectly quiet after food and to refrain from talking. The inclination to coughing should be resisted as far as possible. Various remedies have been recommended for this condition. One of the best is a mixture containing five grains of alum and five minims of liquor potassæ in each dose taken shortly before the meal.

Pyrexia is one of the most important factors in deciding how a patient is to be treated. Considerable surprise and disappointment have often been expressed to me by patients who have been kept in bed after their arrival at a sanatorium. Rest is a sine quâa non when there is pyrexia. But, of course, it makes all the difference whether the rest is obtained in a close, stuffy room or in a pure atmosphere. Hæmoptysis always is an indication for modification of the treatment. The remedies in greatest repute at present are rest and low diet. All cases of profuse hæmoptysis should be kept absolutely at rest, but I do not consider it is necessary or advisable to keep a patient at rest in bed simply because the sputum is slightly coloured with blood. At one time I followed the general rule of keeping all patients with hæmoptysis on low diet, a diet consisting of a limited quantity of fluid in the form of milk and small rations of bread and pounded meat. But except in the case of large and repeated hæmoptysis I do not think the diet is a matter of so much consequence as has generally been taught. Recently I have given up using the special diet for hæmoptysis at the Brompton Hospital and my impression is that patients pass through an attack losing less ground if one feeds them more generously than has hitherto been done.

Treatment requires to be modified according to the stage of the disease. While we consider absolute rest as essential when there is pyrexia we regard exercise to be just as important in the afebrile period. I have already touched on the modifications of treatment called for by such complications as hæmoptysis and dyspepsia. When there is laryngeal tuberculosis rest to the voice is of the highest value. I have been much gratified by the good results obtained in laryngeal disease from the combination of rest to the voice with openair treatment. Far the most difficult cases to treat are those in which there is advanced and active disease. As a rule they must be treated at home. Everything should be done to give them the best possible hygienic advantage to attend to their comfort and to alleviate their sufferings. We must also adapt our treatment to the age, sex, and social position of the patient. Elderly patients, as a rule, do not tolerate cold so well as younger subjects and therefore one hesitates to send them to high altitudes or to recommend the rigcrous treatment of a sanatorium. Fresh air to them is just as important but it needs to be combined with warmth and sunshine. Women I have observed do not respond to sanatorium treatment nearly so well as men. This, I believe, is due to their having, as a rule, less resisting power to the disease and to their being less capable of taking adequate quantities of nourishment. They feel also much more than men the separation from home. It is not only at country sanatoriums that women do less well than men. We find at Brompton Hospital that a smaller percentage of the women are much improved and a larger percentage are not benefited than in the case of the men. Special arrangements may be made for patients to whom money is no object or whose social position and mode of life would make such treatment as is carried out at a sanatorium undesirable if not intolerable.

I propose to say very little about drugs, for time does not permit more than a passing reference to their utility. Medicinal treatment in many cases is of very secondary importance to the hygienic influences I have already considered. It is in the cases that do not do well that we employ drugs most extensively. The hospital patient who leaves much improved will usually be found to have had but. little written on his medicine card. The patient who steadily gets worse does so in spite of the fact that all the resources of medicine are employed on his behalf. The most generally useful medicine is one which will help the patient to eat and aid his digestion. Nothing is better for the ordinary run of patients than the time-honoured mistura gentianæ alkalina of the Brompton Hospital Pharmacopœia taken shortly before food. In some cases an acid, in others an alkali, with gentian, calumba, quinine, or strychnine, may be given. Cod-liver oil is undoubtedly of much value but it is rather a food than a medicine. It has a very high equivalent in calories, a dessertspoonful of the oil being the equivalent of an egg. With regard to those drugs for which something of a specific action has been claimed we may say that they all fail when employed in cases of extensive and spreading disease. Creasote, guaiacol, and their congeners are $\mathbf{n}$ ow much less used than they were a few years ago. Any effect which they have is due to their action on the stomach in stimulating the appetite and thus aiding in the improvement of nutrition. The action of antiseptics administered by the mouth, it is now recognised, is limited to the effect on the digestive tract.

It is in relieving symptoms that the usefulness"of drugs is most apparent. Coumh is the most constant and one of the most troublesome of all the symptoms. Much good may be done by impressing on the patient the importance of restraining cough when it is unattended by expectoration and when it occurs after meals. The cough which occurs in the morning after waking from sleep is one which it is undesirable to check, as it usually results in clearing away the secretion which has accumulated during the night. As a rule ordinary cough linctuses and lozenges should be dispensed with. Tuberculin treatment still finds some adherents and $I$ am in favour of employing it in apyrexial cases which under the best hygienic treatment have improved up to a certain point and then have failed to make further progress. Whether Professor A. E. Wright's tuberculin is or is not an advance on any previous preparation I am not in a position to say. It is only when a treatment is tried on a large scale that any definite pronouncement can be made upon it. Serum treatment has so far proved very disappointing. Professor Marmorek's serum which we lately tried at the Brompton Hospital has proved no more successful in active and progressive cases than others which had been previously put to the test and found wanting.

Whatever plan of treatment is carried out, but especially when hygienic or open-air or sanatorium methods are employed, it is of the utmost importance to secure the intelligent coöperation of the patient. This cannot be done unless we frankly inform him what is the nature of his complaint. In former times it was often the practice to conceal this carefully from him. He was told that his lungs were weak or that he had catarrh or a bronchial attack. To 
overcome the disease it is necessary to give up a good deal of what are ordinarily reckoned the pleasures of life and to implicitly follow out rules of conduct which call for selfdenial and self-control. No one can be expected to do this unless he is satisfied that it is necessary and unless he is possessed of at least ordinary intelligence. One often hears the expression, "the curable stage of phthisis," and I wish to say that I agree with Walshe when he stated that there was no stage of the disease which is surely curable. It is misleading to preach any such doctrine as that consumption is certainly curable if the case is taken in hand early enough. The earlier a case is taken in hand the better but we can never guarantee that a cure will be effected. The most hopeful cases for any form of treatment. are those where the disease is chronic, quiescent, and of small extent. Natural tendency to fibrosis and inherent resistance to the disease are the most important elements which make for recovery. Where the disposition to arrest and repair is strong even the most injudicious treatment may appear of signal efficacy.

In conclusion, let me say that I feel that I have given a very inadequate sketch of the means available for the treatment of consumption. The subject is a very large one and it has been possible only to touch on the most important points. For the hygienic treatment I do not claim that it is the best attainable, only that it is the best so far attained. There is no finality in medicine and the treatment of to-day may be, nay, will be, vastly improved on in the coming to-morrow. However that may be no one, I am sure, will dream of attempting to cure tuberculosis on the old plan of coddling and cosseting patients in the stuffy and vitiated atmosphere which even now is associated in the minds of many as a necessary characteristic of the sickroom.

\section{THE TREATMENT OF STREPTOCOCCIC PUERPERAL FEVER BY ANTITOXIC SERUM.}

BY ALEXANDER G. R. FOULERTON, F.R.C.S. ENG., D.P.H. CANTAB.

LECTURER ON BACTERIOLOGY AND ON PUBLIC HEALTH AT THE MIDDLESEX HOSPITAL MEDICAL SCHOOL; MEDICAL OFFICER TO THE COUNTY COUNCIL OF EAST SUSSEX.

THE uncertainty of the action of antistreptococcic serum in the treatment of streptococcic infections is well recognised in practice and because of it the method of serum treatment has been discredited to a considerable extent in this class of bacterial infections. But the reasons for this uncertainty are easily explained and the complete failure of serum treatment in a large proportion of the cases of streptococcic infection in which it has been used has been inevitable under the existing conditions.

It will be remembered that the first antistreptococcic serum-that of Marmorek-was prepared from a single strain of streptococcus, and it was believed by Marmorek, Behring, and others that the serum thus prepared was specifically antitoxic in all cases of streptococcic infection. And this belief in the unity of streptococcus pyogenes and consequently in the unity of streptococcic toxins and the correlated antitoxins was so firmly held at first that a brand of antistreptococcic serum which was at one time extensively used in this country was prepared from a single strain of streptococcus which was not even derived from a human source but had been isolated from one of the lower animals.

On the other hand, many efforts have been made to differentiate separate species of streptococci and thus streptococcus erysipelatis, streptococcus longus, streptococcus brevis, streptococcus conglomeratus, the enterococcus of Thiercelin, and others, have been described; and there can be but little doubt but that between various streptococci as we isolate them from cases of disease there are often differences - as to their several morphological and cultural characteristics, as to their pathogenic action, and as to their behaviour in relation with different antistreptococcic serums when tested in vitro or by animal experiment-so distinct as to entitle us to assume that we are dealing with definite species of streptococci rather than with mere varieties of a single species, streptococcus pyogenes. And, in short, I : u ppose that at present it would be acknorledged by most bacteriologists who come into actual contact with clinical work that the term streptococcus pyogenes, as we use it, does not denominate a single species but rather is to be understood as a sort of general name which includes several species of cocci, with related varieties, which present a common similarity in their tendency to assume a streptococcal type of grouping. It is, therefore, not a matter for surprise that, applied under such conditions of uncertainty, serum treatment-in itself the most accurately specific method in the whole range of therapeutics and demanding as a necessity equal accuracy in diagnosis-should have frequently proved a failure in cases of streptococcic infection.

The unequal results which had been obtained in clinical practice by the indiscriminate use in every case of streptococcic infection of an antistreptococcic serum prepared from a single strain were soon explained by experiment in the laboratory, and at the same time the way in which the difficulty might be met was pointed out. Amongst much experimental work which has been carried out in the investigation of the question as to the identical specificity or otherwise of different strains of streptococci that of Van de Velde has been perhaps the most suggestive. Van de Velde made a careful study of 21 strains of streptococci derived from the following cases of disease: four from cases of puerperal infection, four from cases of postoperative peritonitis, four from cases of sore-throat, three from abscesses, three from cases of erysipelas, and single strains from cases of arthritis, bronchitis, and cystitis. After a full consideration of the methods of growth and other characteristics of these 21 strains Van de Velde found himself in accordance with the opinions of those who believed in the identical specificity of all varieties of streptococci. But whilst Van de Velde himself came to this conclusion I think that the results of his carefully detailed work are at least open to the opposite interpretation, and for my own part I would claim that his experiments proved such differences between various strains which he worked with as to support what has been already said as to specific differences between some of the strains of streptococci which are found as causers of disease. For the present purpose, however, and in view of the results of Van de Velde's experiments as to the influence of different antistreptococcic serums on different strains this question as to whether the streptococci which he isolated represented one or more species is of merely academic interest and turns mainly upon the precise meaning to be attached to the word "species." Van de Velde carried out a series of experiments in order to test the action of the serum of a horse which has been immunised against one particular strain of streptococcus when used against an experimental infection by another strain. He found that serum of a horse immunised against streptococcus " $\mathrm{A}$," isolated from an abscess, protected to only a slight extent against streptococcus " $P$," isolated from a case of puerperal infection; also it was found that the serum from a horse immunised against streptococcus " $\mathrm{P}$ " did not afford any protection against streptococcus "A," each serum being, of course, actively protective against the particular strain from which it was prepared. A third horse was then immunised against both strains and the serum thus prepared was found to be actively protective against both streptococcus "A" and "P." A fourth antistreptococcic serum (Marmorek's) did not afford any protection against streptococcus " $\mathrm{A}$," and other similar results were obtained with various strains of streptococci. The logical deductions from these results were obvious and Professor Denys of Louvain, at whose suggestion Van de Velde had undertaken his investigation, proceeded to prepare a polyvalent antistreptococcic serum for clinical use, the horse being immunised against strains of streptococci obtained from the most widely diverse forms of disease. And at the present time there are several brands of polyvalent antistreptococcic serum available for general use.

During the years 1896-97-98, when antistreptococcic serum was coming into common use, I gave special attention to the results obtained in the treatment of puerperal fevers, collecting a long series of cases in which one or other brand of antistreptococcic serum had been used. And leaving on one side the cases in which no bacteriological diagnosis had been made, it was obvious that the uncertainty of result which characterised the serum treatment of streptococcic infections generally prevailed also to a very marked extent in the special clinical group of cases of streptococcic puerperal fever. And whilst it was in only a minority of these cases of puerperal infection that serum treatment appeared to have 